\title{
HANdover OPTIMIZATION BASEd ON MEdia INDEPENDENT HANDOVER IN NEW NETWORKS NGWN
}

\author{
Safia Gueziz and Dris Korichi \\ Department of Computing and ICT, Kasdi Merbah University, Ouargla, Algeria
}

\begin{abstract}
One of the challenging issues in Next Generation Wireless Network (NGWN) is achieving seamless Vertical Handover (VHO) while roaming between different technologies such as Wireless Fidelity (Wi- Fi), Worldwide Interoperability for Microwave Access (WiMAX), Universal Mobile Telecommunications System (UMTS) and Long Term Evolution (LTE). F or that the telecommunication operators will be required to develop a strategy for interoperability of these different types of existing network to give the best connection anywhere anytime for the Mobile Users (MUs).

To full these requirements we propose a new VHO approach that based on loose coupling internetworking in conjunction with Mobile IPv4 ( MIPv4) under Media Independent Handover (MIH). Our objective is develop a strategy for interoperability of different technologies and optimized the VHO decision. Our proposed approach provides more exhaustive, high accurate and better performance compared with the approaches proposed in the literature.
\end{abstract}

\section{KEYWORS}

VHO (Vertical Handover); MIH (Media Independent Handover); IMS (IP Multimedia Subsystem); ANSF (Access Network Selection Function); RATs (Radio Access Technologies).

\section{INTRODUCTION}

The next generation wireless networks (NGWNs), called beyond third generation (B3G) or fourth generation $(4 \mathrm{G})$, will include multiple complementary mobile and wireless technologies, all of which will coexist in a heterogeneous wireless access environment and use a common IP core to offer a diverse range of high data rate and multimedia services to end users. Because no single access technology can provide ubiquitous coverage and continuously high quality of service (QoS), multimode mobile terminals will have to roam among the various access technologies to maintain network connectivity and user satisfaction [1]. Therefore, telecommunication operators will be required to develop a strategy for interoperability of these different types of existing networks and required to design of intelligent vertical handoff decision algorithms (VHDAs) for enable Mobile Users to seamlessly get the best connection anywhere anytime.

Two main interworking architectures have been proposed by European Telecommunication Standards Institute (ETSI) namely: loose and tight coupling for integration between the different types of technologies [2]. On the other hand, two main interworking frameworks were proposed by IEEE Group and 3GPP namely: Media Independent Handover IEEE 802.21 (MIH) and IP Multimedia Subsystem (IMS), where each of them requires mobility management protocol and vertical handoff decision algorithms to complement its work $[5,10,12,13]$. 
In this paper we propose a new approach to optimize Vertical handover in Next Generation Wireless Network based on MIH framework. The rest of the paper is organized as follows: section II presents VHO procedure and $\mathrm{MIH}$, in section III we present previous wok related to vertical Handover (VHO) procedure. In section IV we present our proposed approach and finally, conclusion and future work in section V.

\section{Vertical Handover Procedure and MIH}

\subsection{Vertical Handover Procedure}

The mechanism which allows the MUs to continue their ongoing sessions when moving within the same Radio Access Technology (RAT) c overage areas or traversing different RATs is named Horizontal Handover (HHO) and Vertical Handover (VHO), respectively [2]. In the literature most of the research papers have divided the VHO management into three phases: Collecting Information, Decision and Execution [3], [4] as described below.

\subsubsection{Handover Collecting Information}

In this phase, all required information for handover decision is gathered, some of this information is related to the user's preferences (e.g., cost, security), network (e .g., latency, coverage) and terminal (e.g., battery, velocity).

\subsubsection{Handover Decision}

In this phase, the best RAT based on aforementioned information is selected and the handover execution phase is informed about that.

\subsubsection{Handover Execution}

In this phase, the active session for the MU will be maintained and continued on the new RAT. After that, the resources of the old RAT are eventually released.

\subsection{Media Independent Handover (MIH)}

The IEEE Group released IEEE 802.21 standard MIH in 2009 to provide seamless VHO between heterogeneous networks that include both wireless (3GPP and non-3GPP) and wired media [6]. IEEE 802.21 defines two entities: first, Point of Service (PoS) which is responsible for establishing communication between the network and the MU under MIH and second, Point of Attachment (PoA) which is the RAT AP. Also, MIH provides three main services: Media Independent Event Service (MIES), Media Independent Command Service (MICS) and Media Independent Information Service (MIIS) such that MIH relies on the presence of mobility management protocols, such as MIP and SIP, this is shown in Figure 1.

\subsubsection{Media Inde pendent Event Service (MIES)}

It is responsible for reporting the events after detecting, e.g. link up on the connection (established), link down (broken), link going down(breakdown imminent), [6]. 


\subsubsection{Media Independent Information Service (MIIS)}

It is responsible for collecting all information required to identify if a handover is needed or not and provide them to MUs, e.g. available networks, locations, capabilities, cost, etc. [6], this is shown in Figure 1.

\subsubsection{Media Independent Command Service (MICS)}

It is responsible for issuing the commands based on the information which is gathered by MIIS and MIES, e.g. MIH handover initiate, MIH handover prepare, MIH handover commit and MIH handover complete [6].

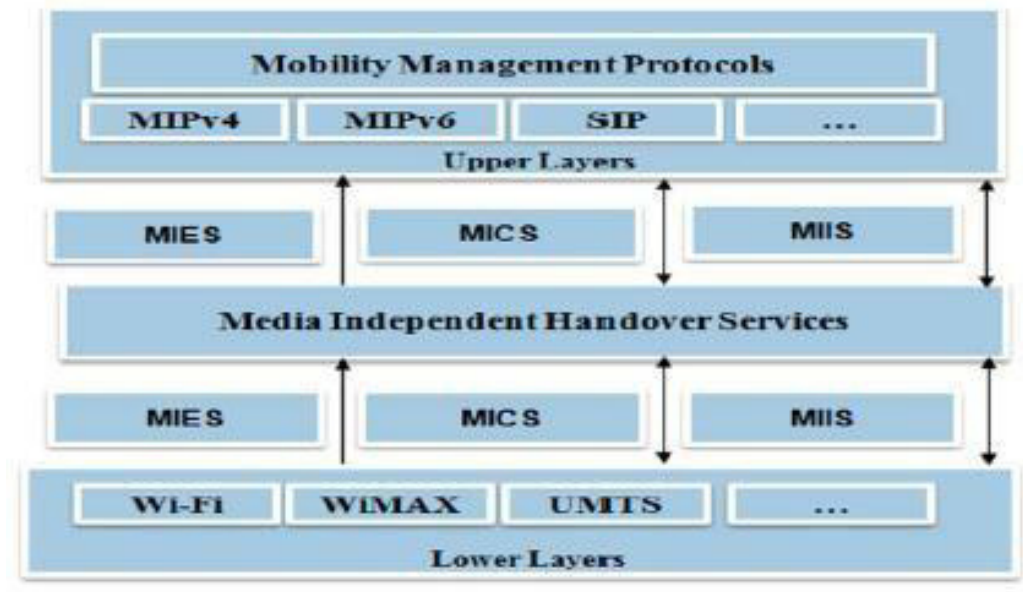

Figure 1. Media Independent Handover (MIH)

\section{RELATED WORKS}

In [6], the authors propose four categories of VHO approaches based on MIH and IP Multimedia Subsystem (IMS) frameworks (MIH based VHO category, IMS based VHO category, MIP under IMS based VHO category and, MIH and IMS combination based VHO category) in order to present their objectives in providing seamless VHO. It has been concluded in [6] that the MIH is more flexible and has better performance providing seamless VHO compared with IMS framework.

In $[11,10]$, The IEEE Group has proposed MIH to provide a seamless VHO between different RATs. The MIH defines two entities: first, Point of Service (PoS) which is responsible for establishing communication between the network and the MU under MIH and second, Point of Attachment (PoA) which is the RAT access point. Also, the MIH provides three main services: Media Independent Event Service (MIES), Media Independent Command Service (MICS) and Media Independent Information Service (MIIS) [11] such that the MIH relies on the presence of mobility management protocols (e.g., MIPv4 and MIPv6).

In [7], the authors propose two categories of VHO approaches based on the mobility management protocols (MIPv4 and MIPv6) which their performances and characteristics have been presented. It has been concluded in [7] that providing service continuity through MIPv4 category under MIH will allow the operators to diversify their acces s networks take into account advantages of this category while MIPv6 category under MIH requires future work improvements in terms of VHO decision criteria, additional entities, complexity, diversity of RATs and evaluation using empirical work real environment. 
And in [8], the authors present the loose and the tight coupling interworking architectures (their objectives, features and challenges). It has been concluded in [8] that the loose coupling is more suitable with MIH and contributes for enhancing its vital role in heterogeneous wireless environment to get fast and soft seamless roaming with minimal latency and minimal packet loss.

In [15], the authors present their approach for enhancing VHO that consists of a procedure which is implemented by an algorithm, but their procedure based only on two different access network for roaming between them (one source network and one destination network) that's not exhaustive procedure in the general cases (in NGWN).

Vertical handover decision involves a tradeoff among many handoff metrics including QoS requirements (such as network conditions and system performance), mobile terminal conditions, power requirements, application types, user preferences, and a price model [1]. The key of VHO management procedure is Access Network Selection (ANS) in the decision phase [15]. There are many proposals introduced by researchers about ANS. A Continues of the studies in [15] the authors in [14], presented their Imperative Alternative MIH for Vertical Handover (I AM 4 VHO) algorithm based on MIH framework and using fuzzy logic to provide low connection failure and low signalling cost. The evaluation shows that the proposed algorithm reduces the probability of VHO connection failure but their access network selection function ( ANSF) based on the same weighting in the different cases of Handoff (imperative, alternative).

\section{OUR PROPOSED APPROACH}

From the previous studies $[6,7,8,9,10,15]$, we propose a new VHO approach that provide an interoperability between differents existing netwoks. Our approach consists of a procedure that based on loose coupling internetworking in conjunction with MIPv4 under MIH . However, no handover decision mechanism is made within MIH [12], "the actual algorithms to be implemented are left to the designers" [13] and implementation of the decision algorithm is out of the scope of MIH [10].

For that we propose a Decision Algorithm that consists of two Access Network Selection Function (ANSFi and ANSFa) for enable the Mobile Users to seamlessly get the best connection in the different cases of Handoff (imperative, alternative), that can provide better performance. Table1 show the comparison between Our Approach and the proposed in [14] and [15].

\subsection{Our Procedure}

We describe our procedure through the VHO phases (Initiation, decision and execution) as depicted in Figure 2.

\subsubsection{Initiation Phase}

While MU is connected to a source network the VHO procedure will be triggered:

- Imperatively due to Radio Signal Strength (RSS) going down or

- Alternatively based on the user's preferences (e.g., high data rate, low cost) [2]. 
International Journal of Computer Networks \& Communications (IJCNC) Vol.8, No.2, March 2016

\subsubsection{Decision Phase}

As a result of triggering in the initiation phase, MIIS Request/Response Available RATs message will be responsible to pass available RATs to MU via source network (PoA and PoS).

- In imperative session due to RSS going down the MU will select RATs list of priority based on our proposed ANSFi Algorithm (case Imperative), and then the MUs pass the RATs list of priority to his source network PoS whereas in,

- Alternative session the MU will select RATs list of priority based on our proposed ANSFa Algorithm (case Alternative) due to his/her profile change, and then the MUs pass the RATs list of priority to his source network PoS.

- When the first choice from RATs list of priority could not be satisfied with available resources (e.g. :the Admission Control AC1 checked the available resources at PoS1 for RAT1 ), the source network PoS will automatically move to another RAT selection in the list in order to satisfy the requirements of this RAT selection and so on.

- Once RAT of sufficient resources has been found, it will be checked by its destination $\mathrm{PoS}$ whether it is compliant with the rules and preferences of operators. If that is available, the MIIS/Home Agent (HA) will be informed to start buffering for new data packets which are sent by Correspondent Node $(\mathrm{CN})$.

\subsubsection{Execution Phase}

In this phase, the MU will be connected to target RAT to start its Authentication, Authorization and Accounting (AAA) with the correspond destination PoA and obtain Care of Address (CoA) from Dynamic Host Configuration Protocol (DHCP).

After that, Update/Ack nowledge binding message notifies HA about the new CoA to start sending the buffered data and continuing the session within ta rget RAT. Finally, after the completion of sending the buffered data the resources is released by MIH [2].

\subsection{Our Algorithm}

Our algorithm based on [14] the authors concentrated on minimization of connection failure and signaling cost which are two vital factors to provide seamless VHO. Our proposed algorithm wich implement our proposed procedure will define two type of VHO (Imperative and Alternative) and defines two Access Network selections Function (ANSFi and ANSFa). The imperative session will be high priority in the case of two VHO sessions in the same time (one imperative, other alternative) [15].

In the imperative VHO case, due to RSS going down the RATs list of priority based on ANSFi will be passed by MU to his source network PoS. When the first choice from the RATs list of priority could not be satisfied with Sufficient of Resources (SoRs) checked by the Admission Control (AC) at correspond destination PoS, the source network PoS of MU will automatically move to the next RAT in the list for satisfying the request and so on, once RAT of sufficient resources has been found, it will be checked by the destination PoS whether it is compliant to the rules and preferences of operators, if that is available, the session will be accepted, otherwise the request will be returned to the source network PoS (of MU) step to select the next RAT in list. Finally, the session will be rejected if there are no available resources for any RAT in the list.

In the alternative VHO case, due to his/her profile change such as data rate, the RATs list of priority based on ANSFa will be passed by MU to his source network PoS, and take the same path of imperative VHO case, as depicted in the Figure 3. 
International Journal of Computer Networks \& Communications (IJCNC) Vol.8, No.2, March 2016

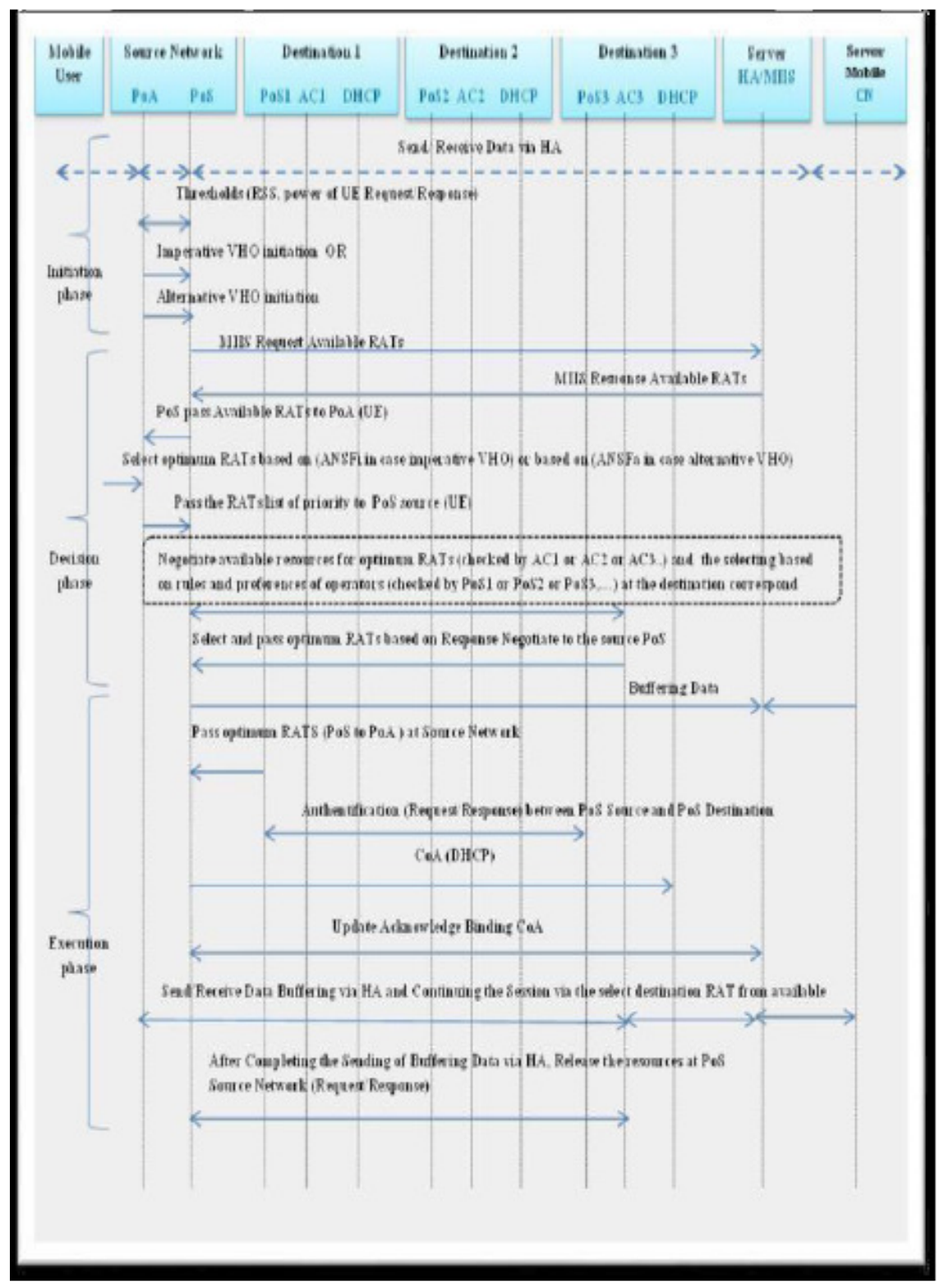

Figure 2. Our Proposed Procedure

\subsubsection{Access Network Selection Function}

Network Selection is the main function of the handover procedure in the Decision phase that is applied when a mobile terminals switches the connection from the Radio Access Networks (RAN) in use to another [16].

Based on the information collected in the Initialization Phase that is necessary for decision making regarding the selection of the best access network. The considered criteria takes into account in ANSF are both user preferences and network status. 
Our Approach defines two Access Network selections Function ANSFi and ANSFa for Impérative, Alternative case respectively. In Impérative case ANSFi select the RAN by considering only one performance metric that is Received Signal Strength Indicator (RSSI), measured by MN. In Alternative case ANSFa consider multiple metrics, simultaneously, to select the RAN to be employed (RSSI, Monetary cost, Battery lifetime, MT velocity, Network load).

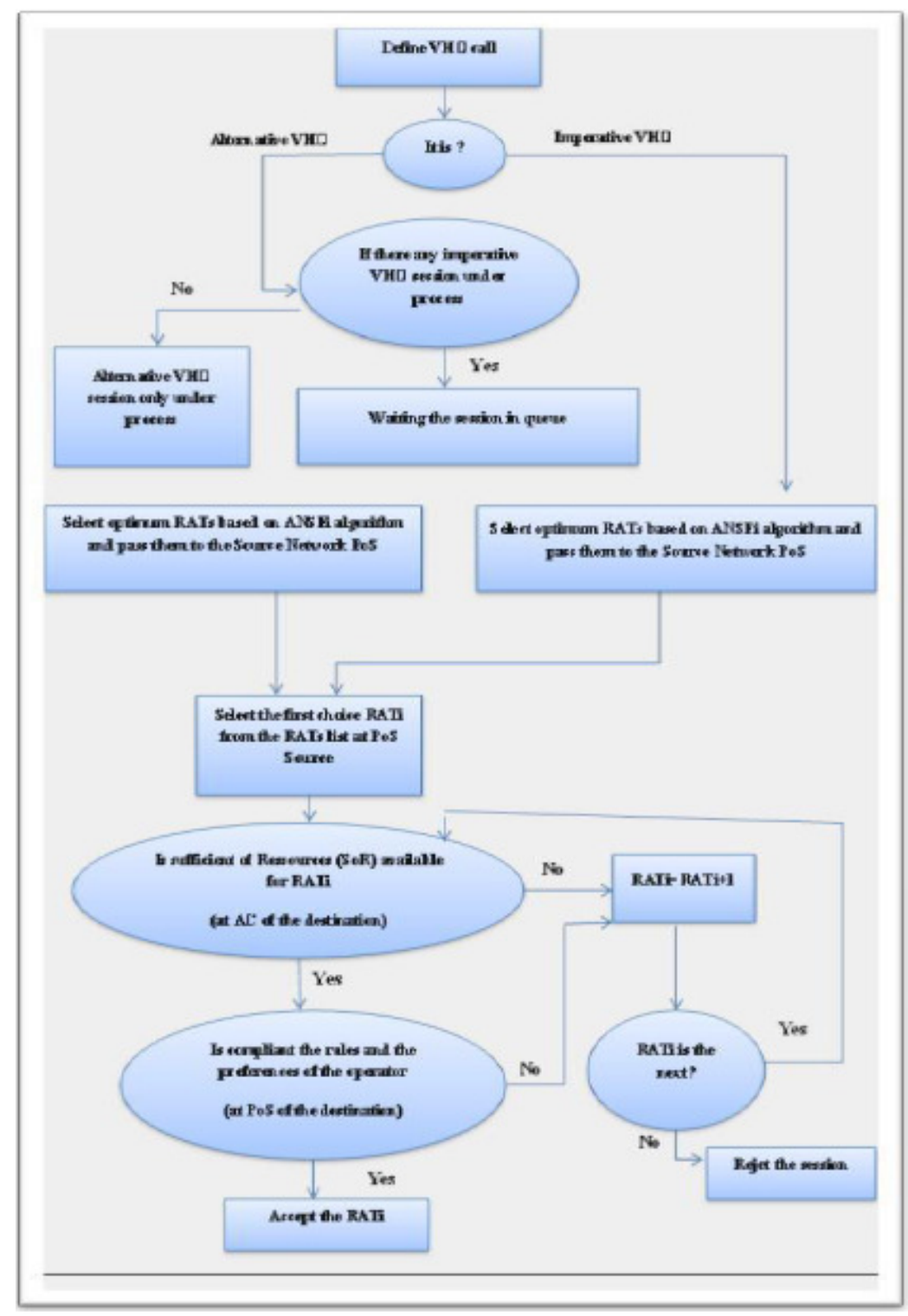

Figure 3. Our Proposed Algorithm 
International Journal of Computer Networks \& Communications (IJCNC) Vol.8, No.2, March 2016

Table 1. Comparison between our approach and the proposed in $[14,15]$.

\begin{tabular}{|l|l|l|}
\hline Approach & Exhaus tive & Accurate of the approach \\
\hline In [13,14] & $\begin{array}{l}\text { Less due to: } \\
\text { The procedure based only } \\
\text { on two different access } \\
\text { network(source and } \\
\text { destination) }\end{array}$ & $\begin{array}{l}\text { Moderate due to: } \\
\text { Using the same Access Network Selection } \\
\text { function in the two case: Imperative VHO and } \\
\text { Alternative VHO }\end{array}$ \\
\hline $\begin{array}{l}\text { Our } \\
\text { approach }\end{array}$ & $\begin{array}{l}\text { More due to: } \\
\text { Our procedure based on } \\
\text { many different access } \\
\text { network (more compliant } \\
\text { with case general in } \\
\text { NGWN) }\end{array}$ & $\begin{array}{l}\text { Usigh due to: } \\
\text { Function, (ANSFi) in the case imperative VHO } \\
\text { and (ANSFa) in the case alternative VHO }\end{array}$ \\
\hline
\end{tabular}

\section{CONCLUSIONS}

In this paper we presented our VHO approach based on the approaches that have been studies in the literature with more exhaustive, high accurate and better performance for enhancing VHO procedure and VHO algorithm compared with the previous works. The proposed VHO procedure based on loose coupling conjunction with MIPv4 under MIH to execute our procedure as depicted in Figure3. Also, our procedure achieves less connection failure due to using the optimum RATs (list of priority) based on our ANSF (ANSFi and ANSFa) as depicted in Figure4. In future work we plan to execute our proposed Approach by considering different scenarios and by different methods of experimentation (Simulation/Emulation) to evaluate the performance of our approach.

\section{REFERENCES}

[1] Y.Nkans ah-Gyekye, J.I. Agbinya, (Nov 2008), "A Vertical Handoff Decis ion Algorithm for Next Generation Wireles s Networks ". In: Third International Conference on Broadband Communications, Information Technology \& Biomedical Applications, pp.358-364, 23-26.

[2] O.Khattab, O. Alani, (Jun 2014), "Simulation of Performance Execution Procedure to Improve Seamles s Vertical Handover in Heterogeneous Networks ". In: International Journal of Advanced Computer Science and Applications (IJACSA), pp. 109-113, vol. 5, no. 6.

[3] M. Zekri, B. Jouaber, D. Zeghlache, (Oct 2010), "Context Aware Vertical Handover Decision Makingin Heterogeneous Wireless Networks". In: 35th Conference on Local Computer Networks 2010 (LCN 2010), pp. 764-768, 10-14.

[4] M.Kassar, B. Kervella, G. Pujolle. (Jun 2008), "An overview of vertical handover decisions trategies in heterogeneous wireles s networks ". Computer Communications, vol.31, no. 10, pp.

[5] O.Khattab, O. Alani, ( Jul 2014),"The Des ign and Calculat ion of Algorithm for Optimis ing Vertical Handover Performance". In: 9th IEEE/IET International Symposium on Communication Systems, Network s and Digital Signal Processing 2014 (CSNDSP 2014), pp. 421-426, 23-25.

[6] O.Khattab, O. Alani. (Jun 2013), "A Survey on M edia Independent Handover (MIH) and IP Multimedia Subs ys tem (IMS) in Heterogeneous Wireles s Networks ”. In: International Journal of Wireless Information Network s (IJWIN), Springer, vol. 20, no. 2, pp. 215-228.

[7] O.Khattab, O. Alani, (Apr 2013), "Survey on Media Independent Handover (MIH) Approaches in Heterogeneous Wireless Networks”. In: 19th European Wireless 2013 (EW 2013), pp. 1-5, 16-18. 
[8] O.Khattab, O. Alani, (Jul 2014), “An Overview of Interworking Architectures in Heterogeneous Wireles s Networks : Objectives, Features and Challenges ". In: 10th International Network Conference 2014 (INC 2014), pp. 71-79, 8-10.

[9] P.Neves , J. Soares , S. Sargento, (Dec 2009) "Media Independent Handovers : LAN, MAN and WAN Scenarios ”. In: IEEE GLOBECOM Work shops, pp. 1-6.

[10] G.Lampropoulos, A.K. Salkintzis, N. Passas,(Jan 2008), "Media Independent Handover For Seamless Service Provision in Heterogeneous Networks". IEEE Communication Magazine, vol. 46, no. 1, pp. 64-71.

[11] J.Marquez-Barja, C.T. Calafate, J.C. Cano, P. Manzoni. (Mar 2011), "Evaluation of a Technology Aware Vertical Handover Algorithm Based on the IEEE 802.21 Standard". In: Wireless Communications and Networking Conference 2011 (WCNC2011), pp. 617-622, 28-31.

[12] S.Frei, W. Fuhrmann, A. Rinkel, B.V. Ghita. (Feb 2011), "Improvements to Inter System Handover in the EPC Environment". In: 4th IFIP International Conference on New Technologies, Mobility and Security 2011 (NTMS 2011), pp. 1-5, 7- 10.

[13] X.Yan, Y. Ahmet Şekercioğlu, S. Narayanan,(Aug 2010), "A Survey of Vertical Handover Decision Algorithms in Fourth Generation Heterogeneous Wireless Networks ". Computer Networks , vol. 54, no. 11, pp. 1848-1863, 2 Aug 2010.

[14] O.Khattab, O. Alani, (Aug 2014), “Algorithm for Seamless Vertical Handover in Heterogeneous Mobile Networks". In: IEEE Technically Co-Sponsored Science and Information Conference (SAI 2014), pp. 1-8, 27-29.

[15] O.Khattab, O. Alani,(Jun 2012), "Improvements to Seamless Vertical Handover between Mobile WiMAX, Wi- Fi and 3GPP through MIH”. In: 13th Annual Post Graduate Symposium on the Convergence of Telecommunications, Networking and Broadcasting 2012 (PGNET 2012), pp. 31$35,25-26$.

[16] I.Bisio, S. Delucchi, F. Lavagetto and M. Marchese. (Jan 2015), "Performance Comparis on of Network Selection Algorithms in the Framework of the 802.21 Standard". JOURNAL OF NETWORKS, VOL. 10, NO. 1.

\section{AUTHORS}

Safia Gueziz born in 1989 in Ouargla, Algeria, a graduate of Bachelor in Computer Studies from the University kasdi merbah in 2010, had awarded here master degree in computer engineer in 2012. She is Phd Stududant in computer systems and networks in the same university.

Dr. Dris KORICHI was born in Ouargla, Algeria on December 10th 1969. He obtained his Ph. D. from Paris XI university, France on 2000. Currently, he is an Associate Professor at the Computer and ICT department at the University of Ouargla, Algeria.
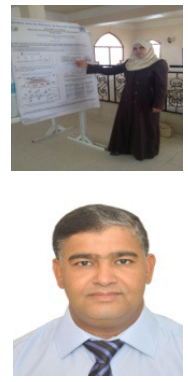